\title{
Sony Labou Tansi : L'Anté-peuple ou le peuple hanté
}

\section{Patrice Yengo}

URL : http://journals.openedition.org/coma/520

DOI : $10.4000 /$ coma.520

ISSN : 2275-1742

\section{Éditeur}

Institut des textes \& manuscrits modernes (ITEM)

\section{Référence électronique}

Patrice Yengo, «Sony Labou Tansi : L'Anté-peuple ou le peuple hanté », Continents manuscrits [En ligne], 4 | 2015, mis en ligne le 15 mars 2015, consulté le 16 avril 2021. URL : http://journals.openedition.org/ coma/520 ; DOl : https://doi.org/10.4000/coma.520

Ce document a été généré automatiquement le 16 avril 2021.

\section{(c) (i) () $\Theta$}

Continents manuscrits - Génétique des textes littéraires - Afrique, Caraîbe, dispora est mis à disposition selon les termes de la licence Creative Commons Attribution - Pas d'Utilisation Commerciale - Pas de Modification 4.0 International. 


\title{
Sony Labou Tansi : L'Anté-peuple ou le peuple hanté
}

\author{
Patrice Yengo
}

1 Sur Sony Labou Tansi, bien des choses ont déjà été dites et quelquefois des plus farfelues. Il n'est qu'à lire les gloses des soi-disant « experts » de la littérature africaine sur les «petites mains » de Sony Labou Tansi pour s'en convaincre. Or ce qui frappe d'emblée lorsqu'on se penche sur l'œuvre de cet écrivain c'est son caractère extrêmement prolixe : sept romans, huit pièces de théâtre, des nouvelles, des recueils de poésie, et, seize ans après sa mort, nous n'en avons pas encore fait le tour. La publication il y a quelques années par la Revue noire du coffret L'Atelier de Sony Labou Tansi $^{1}$ en est la preuve. Et depuis que je travaille dans l'équipe de recherche "Manuscrits francophone» de l'ITEM, aucun jour ne passe sans que nous nous extasiions, mes collègues et moi, sur les joyaux que nous ne cessons de découvrir dans les cartons inexplorés des manuscrits de Sony.

2 Sony Labou Tansi - Marcel Sony de son état-civil-, est né en 1947 en République démocratique du Congo et est décédé le 14 juin 1995 à Brazzaville. Scolarisé d'abord en kikongo au Congo-Kinshasa où il a vu le jour, il a poursuivi ses études au CongoBrazzaville d'où sa famille était originaire; il est devenu par la suite professeur d'anglais. Considéré comme le chef de file d'une nouvelle génération d'auteurs francophones d'Afrique noire, il a créé le Rocado Zulu Théâtre à Brazzaville qui lui a permis de mettre en scène l'ensemble de ses pièces. Son œuvre a été consacrée par de nombreux prix dont le Grand prix littéraire de l'Afrique noire pour L'Anté-peuple ${ }^{2}$ en 1988.

\section{Comment résumer ce livre?}

3 L'Anté-peuple est son premier roman. Il a été écrit en 1976 et publié seulement en 1983 à la suite du «légendaire » La Vie et demie et du scandaleux L'État honteux. Mais avant de donner notre résumé du livre, voici ce que l'auteur disait lui-même de son livre à 
Pierrette Herzberger-Fofana de la Friedrich-Alexander-Universität ErlangenNürnberg :

Le fond du roman repose sur une histoire vécue. J'ai gardé le nom de Dadou, le personnage principal qui vit actuellement à Brazzaville. À la suite d'un faux témoignage contre lui, il a dû s'évader de sa ville natale, Kinshasa. Car il était accusé d'avoir terni l'honneur d'une jeune fille. Et cette dernière s'est donc retrouvée enceinte. J'ai été choqué de voir qu'aucun moyen scientifique n'a été utilisé pour établir la paternité biologique. Ce genre de situation bâtarde relève plus de l'émotion que de la raison pure et porte ainsi souvent préjudice à des innocents. Dans le roman, Yavelde est amoureuse de son professeur, ce qui n'est pas interdit, mais elle est désespérée et commet un geste incontrôlé : elle se suicide. Le geste d'un être mû par la passion. Tel est le récit romancé. Quant à la vraie histoire, elle m'a été racontée en 1977 à Pointe-Noire par un jeune homme que j'ai rencontré chez mon ami André. Ce jeune homme sympathique dormait sur une natte au salon. Le titre initial du roman d'ailleurs était La Natte. J'ai donc été surpris de voir que cet être posé, intelligent et humain, dormait par terre en pleine ville. Je lui en ai demandé la raison. Il m'a expliqué qu'il avait été obligé de fuir Kinshasa à cause d'une fausse accusation: une jeune fille savait qu'il était innocent, mais ne pouvait pas le prouver. À partir de cette anecdote, j'ai créé les personnages de mon roman et toute l'action qui en découle ${ }^{3}$.

Le « héros » de L'Anté-peuple, le citoyen Dadou, est directeur de l'école normale de filles à Kinshasa. Résistant aux avances d'une jeune élève, Yavelde, il se trouve entraîné dans la spirale d'une passion obsessionnelle à laquelle il essaie d'échapper en buvant. Non seulement, il n'y arrive pas mais ce «citoyen " respectable tombé petit à petit dans l'ivrognerie et le déshonneur doit désormais faire face à un " complot » post mortem. En effet, la jeune fille s'est tuée de désespoir en laissant une lettre qui accuse Dadou de l'avoir mise enceinte. À cette nouvelle, la foule saccage sa demeure. Ses deux enfants sont lynchés et sa femme se donne la mort de désespoir. Arrêté, il est jeté en prison. Au bout de quatre ans d'incarcération sans jugement, il arrive à s'échapper grâce à des complices qui l'aident à passer de l'autre côté du fleuve où il trouve refuge dans un village de pêcheurs. Dadou y est à nouveau arrêté. Cette fois, ce sont les hommes de la milice du Parti, les « Bérets », qui l'emprisonnent. Torturé et laissé pour mort dans un ravin, il est sauvé in extremis par des maquisards. Le voilà enrôlé dans le maquis et obligé d'errer à Brazzaville sous le déguisement d'un fou. C'est la défroque de cette folie simulée qu'il commet l'attentat que tout le monde attendait en mitraillant le Premier secrétaire du Parti en pleine messe de Pâques. Entretemps, il est rattrapé par une autre femme, Yealdara, cousine de la première, qui l'a suivi de Kinshasa et a fait le même chemin que lui. C'est auprès d'elle qu'il retrouve son cœur d'enfant tandis que tous les fous sont pourchassés et exécutés dans la ville de Brazzaville. Dès lors, l'existence redevient aléatoire car « (...) si la vie cesse d'être sacrée, la matière, toute la matière ne sera plus qu'une sourde folie ${ }^{4}$. » La folie est peut-être le maître mot de cette œuvre étonnante, détonante, car dans " ce pays que Dieu a quitté », la folie est ce contre quoi tout le monde lutte. C'est elle qui imprègne de sa surréalité tous les personnages, les évènements et l'histoire même qui, pour le coup, compte peu. Mais, contre elle il y a le foisonnement de la vie au-delà même de la vie misérable de la mort par contumace.

\section{Folie et mort : un peuple hanté}

5 Mais pour coller au plus près à cette folie, il faut s'éloigner des berges de l'exotisme. Pas de gris-gris, de féticheurs, de scènes de sorcellerie. Pas de folklore. Nul souci de plaire 
non plus. L'Anté-peuple, c'est donc avant tout le regard lucide, combattif et sans concession que l'écrivain porte sur l'Afrique postcoloniale et particulièrement les deux Congo hantés par deux dictatures qui, pour se réclamer de deux idéologies différentes, ne relèvent pas moins du même envers où les oligarchies prennent possession des États-nations bricolés par les anciens colons belges et français. Sony Labou Tansi est lui-même victime de ce bricolage, puisque natif du Congo belge, qui se changera en Zaïre sous Mobutu avant de redevenir Congo (République démocratique du Congo), il fera ses études et enseignera au Congo-Brazzaville. Mais l'attaque porte moins sur les anciens colons, que sur les dirigeants actuels, ceux qui ont hérité du pouvoir et qui d'une rive à l'autre ne cessent de fomenter des coups d'État, des guerres sans fin. Aucune discontinuité donc entre des dictatures qui sont parfaitement transposables jusque dans leurs rites de pacotille, mais aucune discontinuité non plus du côté du génie du peuple et de son identité. C'est grâce à ce génie commun que d'un côté ou de l'autre du fleuve, l'on se reconnaît et s'entraide.

Mais le peuple hanté ne l'est pas seulement par ses dictateurs. Il l'est aussi par ses propres démons, héritages peu consensuels d'un monde en mouvement qui n'a réussi à battre en brèche l'ordre ancien que pour mieux exalter les fantasmes individuels. Et la femme est au centre de ce fantasme. Loin de la figure de victime, mineure et exploitée, qu'on aime à lui assigner en Europe, la femme africaine, congolaise, est ici aux deux bouts de la chaîne de la narration. Ange de la tentation, elle est la source des malheurs du héros dont elle réveille les désirs les plus enfouis, mais elle est aussi, par la grâce de l'amour, celle par laquelle la rédemption arrive. Paradoxalement, c'est par la vertueuse Yavelde, dont le nom évoque le mot Yaveh, que le scandale surgit, alors que Yealdara, fille libre, sacrifie sa liberté pour lui. C'est que Yealdara est " peut-être, comme toutes les femmes, putain, mais putain des hautes sphères morales, putain du large de la vie ; et le large de la vie, pour y aller, il faut du talent, de la patience ${ }^{5}$.»

7 Le héros, qui se tient entre ces deux pôles de l'image - presque stéréotypée - de la femme, n'est pas un homme d'exception, loin s'en faut. Bien qu'il ait été un ancien étudiant de l'Université Lovanium (Kinshasa) et qu'il ait soutenu une thèse en droit sur L'Ombre de la loi chez les criminels, cet ancien partisan de Lumumba qu'est Dadou, esprit en apparence scrupuleux, n'est qu'un personnage falot, sans grande envergure, qui traîne son mal-être comme un fardeau au gré des événements. Les premiers, qui donnent prétexte au roman, sont pour le moins mesquins voire insignifiants. Et les néologismes bâtards comme merdant ou les qualificatifs auto-dépréciatifs comme moche, qu'il applique aussi à tout ce qui l'entoure, finissent par le rattraper. Lorsque dans la deuxième partie du livre, il se dilue dans le paysage pour faire place au personnage féminin de Yealdara, il n'y a pas de place alors que pour la "mocherie ", une "mocherie " gluante qui s'incruste dans toute la société, celle des humbles brisés par la terreur comme celle des puissants et des hommes forts, égarés dans leur folie meurtrière : «Il y a ici la naissance d'un phénomène qui devient progressivement naturel et qui s'appelle "mocherie". Et c'est pourquoi nous vivons et crevons dans ce monde le plus moche du monde ${ }^{6}$. » À ce niveau, plus rien ne résiste à la mocherie, qui devient collective et caractérise désormais ces êtres de l'entre-deux-mondes, partagés entre l'inquiétude quotidienne et la peur panique, l'absurde et l'horreur, que Sony Labou Tansi désigne par le terme des "peut-être-vivants »" Pourtant, dans cette vie approximative, immergée dans une totale atmosphère de cauchemar, où le meurtre est quotidien et la vie dévaluée, il est encore question de vie. D'abord parce que ces deux 
pays, les Congo, qui irriguent ce récit sont paradoxalement des «pays où les choses sont les plus tendres du monde. Le ciel, le fleuve, l'herbe - tout est tendre. Mais c'est sur cette divine tendresse des choses que les hommes se tuent ${ }^{8}(. .$.$) . » Ensuite, parce que$ Sony sait rendre tragique toute sa poésie, au-delà de l'absurde.

Cependant, le tragique dans sa dimension poétique ne tient pas exclusivement à l'histoire mais à la façon de la raconter. La narration chez Sony Labou Tansi est fondée sur l'idée qu'il faut envisager le récit en immersion dans son contexte social et politique, dans son environnement intérieur et extérieur. Défilent alors sous la plume de l'auteur une multitude de personnages aussi singuliers que visuellement contrastants. Citons pour mémoire, outre Dadou, Yavelde et Yealdara, du côté de Kinshasa : le commissaire, Landu le chauffeur, Malvoisi avocat et ancien professeur de droit, Falodiati compagnon de cellule de Dadou emprisonné pour détournement de fonds, le régisseur de la prison etc. De l'autre côté du fleuve, l'on va à la rencontre de Fortuné Loupanzo, un vieux qu'on appelle aussi Sacramento, de Sylvain et Henri, de Mpené Maléla, d'Abounkira, de Zarathoustra ou de la folle Kaounsura etc. La description qui accompagne chacun des personnages rompt presque avec la fiction et flirte avec une authenticité documentaire qui déborde le récit comme dans cette appréciation qui est faite du chauffeur Landu : "Son âge témoignait et sa manière de combiner la politesse à la politique. Politisé et poli, (...) Landu disait ses «M'sieur le Citoyen » avec un indice de vénération.»

\section{Chronique urbaine et réalisme quotidien}

Dès ce moment-là, le récit prend l'allure d'une chronique urbaine et fait un sort à certains lieux, surtout ceux fréquentés par les élites, «Le Magistrat», "Le Quatredeux-quatre, le night-club de l'Intelligentsia » ou «Chez tantine Kamikaze ». Nous nous familiarisons avec les quartiers de la ville de Kinshasa, Matongué, Limété, les deux Yolo : Yolo-Nord et Yolo-sud, Ranquin, Kalamu. Même les rues se déclinent dans leur particularisme hérité de la colonisation belge: «rue Kabambala numéro septantedeux $»^{10}$. Cette chronique nous ouvre les portes d'un univers urbain complexe où le quotidien s'extériorise en marques de voitures, «Datsun, La vieille Mazda, Peugeot 404 » ou de bières, «Polar, Amstel, Heineken, Régla, Skol», en marques de produits alimentaires, «Lait Nido », ou d'habillement, la veste « Abacost » imposée par Mobutu comme symbole de l'authenticité zaïroise. Mais, malgré son naturalisme, le roman déroule la représentation d'une réalité qui est de loin indépendante du réel qu'elle décrit: "Les lampes trop espacées accusaient une fatigue, un curieux désespoir ${ }^{11}$. " Chacun y lutte pour se faire entendre, être reconnu et réussir, c'est l'article 15. C'est le monde de la débrouillardise : «On disait que l'homme d'ici était cent fois plus malin que son salaire ${ }^{12}$.»

Le déroulé des noms n'est pas une simple litanie des lieux et places à usage touristique mais un acte de nomination. «En tant qu'écrivain, mon travail consiste à nommer (...) Et je crois que dans tout ce que j'ai écrit, j'ai nommé ", disait Sony Labou Tansi en substance. Car nommer une chose, c'est la faire advenir, la rendre sensible et intelligible, l'insérer dans un ordre où elle acquiert toute sa teneur symbolique en se dévoilant. L'écriture vient ici en renfort de cette culture de l'oralité et de l'incarnation, qu'on peut appréhender à partir des chansons du musicien-compositeur Tabu Ley (alias Rochereau) dont il assure la traduction (Mokolo na koKufa) ou triture les textes : « Banda 
yangaï bomwana / nazwaka te kaka Nzambé / nako kwamisa. Il avait changé le dernier mot du couplet pour mettre la chanson à sa propre dimension - Ley avait parlé de "prier Dieu" [ko sambela], mais lui alla trouver le mot kwamisa. Dans une des langues du pays: emmerder Dieu, c'est plus humain - c'est moins moche, et Dieu doit aimer ça ${ }^{13}$. »

11 Mais nommer, c'est aussi certifier d'une occurrence et l'inscrire dans la chaîne signifiante de la lignée familiale comme marqueur patronymique :

- Si on fait un gosse, on l'appellera Natty, ou peut-être Natta.

- Ça ne veut rien dire, Natty.

- Ça vient de la natte [...], je pense à ma natte, là-bas en prison ${ }^{14}$.

12 Subtile référence au titre original de ce livre qui devait s'appelait au départ La Natte et à la façon qu'ont les Bantous de prénommer leur progéniture - natta voulant dire aussi porter. Cette utilisation des mots peut aussi opérer un transfert de la puissance des ancêtres ou des disparus sur les vivants "Sadi-Motara était le nom d'un oncle à moi, très célèbre. J'espère qu'il vous portera bonheur ${ }^{15}$." car dans ce pays, « Il y avait des noms qu'on versait comme du vin sur la tête des choses. Et ça se mêlait. Et ça dégageait l'espoir ${ }^{16}$.» L'espoir est bien l'horizon de cette tragédie contre laquelle il faut imposer la vie et ses mots mais surtout ce qui git sous les vies et les mots: «La vie. C'est toujours lourd. Les mots aussi. Mais maintenant, ce qui compte pour moi c'est ce qui dort sous les mots (...) ; ce qu'il y a sous les vies ${ }^{17}$ ». Il se trouve que sous les mots, une matérialité se rebelle à être désignée; des éléments, des choses se moquent du nom qu'on leur donne, se situant au-delà des mots, de toute éternité : « Majestueux fleuve ! Nous t'appelons Congo. Nous t'appelons Zaïre, mais toi, comment tu nous appelles? Comment tu nous vois ? Comment tu nous penses ${ }^{18}$ ?».

Dans ce milieu tout change à grande vitesse. Les ministres défilent au gouvernement au gré de l'humeur du père de la nation et guide providentiel, les rues, les places publiques sont débaptisées à tour de bras. Et même dans la vie quotidienne, une danse en remplace une autre au rythme infernal des succès musicaux des orchestres de Kinshasa: "Le "choquer" était cette nouvelle danse zaïroise qui avait détrôné la "cavacha" et l'"ekonda saccadé"19." Il faut savoir garder en mémoire et nommer devient l'acte par lequel l'actualité s'instruit de la longue chaîne de la réminiscence. Preuve de l'absurdité des changements préconisés en Afrique, celui des noms que Mobutu avait imposé en substituant les noms « authentiquement zaïrois » aux prénoms chrétiens ou musulmans. Ces changements qui stipulaient clairement qu'on « n'a pas besoin “d'anciens hommes" "20 portaient tous l'ambition de créer «l'homme nouveau »- fin mot des indépendances - : «Un seul mot avait frappé les oreilles de Dadou : ancien homme. Il y avait du vrai là-dedans ${ }^{21}$. » Mais dans ce jeu de substitution, l'authenticité zä̈roise, héritière de la toute-puissance coloniale, se double des lubies de nouveaux dirigeants. Yealdara doit affronter le harcèlement d'un agent de «la Commission spéciale du protocole authentique. Il cherche des jolies pour les présidents qui viennent aux assises de l'OUA. Ça fait deux jours qu'il me court après. Il veut ramener un bon morceau pour avoir une bonne enveloppe ${ }^{22}$.»

\section{Au cœur du tragique mais au-delà de la mort}

14 Il apparaît clairement que la tragédie s'assume, jusqu'à l'excès, dans la performance mécanique des nouveaux cultes et de ses rites. Et les papiers sont les nouveaux fétiches de cette religion de « l'homme nouveau » : « Il n'y a plus que les papiers qui raisonnent, 
qui pensent, qui respirent ${ }^{23}$.» « (...) la carte de visite, puis la vieille carte nationale, puis la carte de dévouement à la cause, puis la carte du Parti sur celle du Rassemblement des femmes $»^{24}$. Mais contre les papiers, il y a la vie et l'homme de chair, le Noir qui « est plus malin que les papiers. Il les aura toujours ${ }^{25}$. » Il faut croire que personne n'est dupe même si et surtout si l'on joue à le paraitre: «Ici, être dupe devenait souvent le bonheur le plus sûr et le plus grand ${ }^{26}$." Car si la survie est dans le faire-semblant, le salut est dans la croyance. Toutefois la croyance est ailleurs: "Ici, nous croyons fortement, nous croyons en tout. Nos idées font ce que nos corps ne peuvent pas $^{27}$. » En fait ce que les corps ne peuvent pas faire c'est à Dieu qu'il est demandé de le faire: "Seigneur Dieu, souffla la vieille, fais ta part des choses. Nous avons fait la nôtre ${ }^{28}$." Chez Sony Labou Tansi, la supplique vient révéler une exigence de révolte contre l'inertie générale et un acte de rébellion qui s'édifie dans le meurtre du Premier secrétaire du parti, assassiné à la messe de Pâques, et dont la ritualité tragique est transformée en une esthétique du désespoir. Dadou en réchappe, certes mais cela a-t-il vraiment une importance? Puisque personne ne réchappera, pour sûr, de cet engrenage infernal.

L'assassinat du dirigeant est l'aboutissement d'un texte qui institue ainsi la cruauté comme règle d'or de l'absurdité et de l'arbitraire postcolonial. Il s'opère par la même occasion un déplacement du sens des mots; de la mort sur la vie et l'engagement. Le chef qui confie à Dadou la mission de tuer le Premier l'énonce plus ou moins explicitement: "Nous sommes des morts. Et un mort ne pose pas de questions. Un mort, ça pourrit. Vite ou lentement, mais ça pourrit. Les questions, les réponses, nous, on les laisse aux vivants ${ }^{29}$. " Dès qu'il est passé de l'autre côté du fleuve, Dadou est conscient qu'il a rejoint les rives des morts, celle des gens du fleuve qui ont refusé une appartenance sociale faussée par les compromissions et ont décidé de mourir pour renaître à la vraie vie.

La première tâche qui s'impose, pour ne pas avoir à choisir entre une mort « de mouche » et une mort « d'homme », est d'apprendre à apprivoiser la mort, à " mourir vivant", comme le dit Sony Labou Tansi lui-même dans un entretien avec Guy Daninos: «Je sais que je mourrai vivant. Tous les hommes devraient mourir vivants. C'est si beau. » La mort ainsi démystifiée ne guérit pourtant pas de la frayeur qu'elle suscite. Elle ordonne même de recentrer le questionnement sur le sens à lui donner dès lors que la vie elle-même devient insupportable: «Je ne sais pas à quoi ressemble la mort : à un fleuve ? à un pont ? à un mur ? à une porte ? J'en ai peur. Mais j'ai plus peur de la vie, cette vie où je laisse un monstre exécrable: Nitu Dadou ${ }^{30}$.» Il n'est plus d'autres choix que la mort prenne la signification de la rédemption afin qu'elle puisse donner lieu à l'engendrement d'autres existences.

Mais avant d'accepter cette belle mort, les personnages de Labou Tansi doivent subir la solitude et le rabaissement. Dans la seconde partie de L'Anté-peuple, Dadou, ayant perdu sa dignité, passe ses nuits dans les caniveaux, avec pour matelas les eaux stagnantes et pour taie d'oreillers les immondices. Le miséreux forme corps avec la boue, c'est-à-dire la Terre. Chez Sony Labou Tansi, les nombreuses scènes de tortures et de dissections attestent du peu de crédit accordé au corps humains et de l'humiliation qu'on lui fait subir. Elles établissent un rapport entre la condition des forçats et celle des morts, mais aussi un rapport entre le corps et la Terre. 


\section{Corps, chair, viande : les métaphores du corps} privilégiée au pouvoir. Pouvoir de séduction, cela va de soi : «Le corps de cette gamine lui parut contagieux $(. . .)^{31}$. ", mais aussi avec la mémoire pour devenir une composante essentielle à la fois de la poétique et de l'éthique : «L'orchestre exécutait une rumba. Longue, cette rumba ; les couples se dilataient, perdus dans l'enchevêtrement des sons. Longue, longue cette rumba. Longs, amples et tendus, les corps ${ }^{32}$.» Qu'il soit réel ou symbolique, c'est à travers le corps et par lui que circulent les éléments constitutifs du texte et que s'organise le récit : « La loi. Elle n'était pas moche, la loi. Le corps, oui. Tous les corps sont moches. D'ailleurs, à bien y réfléchir, le corps, c'est le sommet de la "mocherie". La preuve, eh! bien la preuve, la grande preuve c'est qu'il ne se lève que pour tomber ${ }^{33}$. " L'analogie que l'auteur établit entre corps et sexe masculin (qui ne se lève que pour mieux retomber) relève d'un jeu de mots subtil emprunté aux langues Kongo. Elle est caractéristique de ces écritures africaines qui, se jouant du bilinguisme, cherchent à établir des passerelles entre les langues vernaculaires et la très officielle langue française. En jouant souvent des néologismes à partir du français lui-même: «merdant, merdé, s'enconchonner, blanconnerries », ou en détournant une locution bien connue "Il y a anguille sous pirogue, il y a anguille sous béret", voire en traduisant certaines propositions comme « avoir deux cœurs » qui exprime l'embarras d'une personne confronté à un dilemme. De même lorsque Dadou dit «J'ai eu lieu, tu peux me croire sur parole : j'ai eu lieu ${ }^{34}$.", Sony Labou Tansi traduit tout simplement une expression congolaise dans laquelle avoir eu lieu signifie qu'on a eu une existence sociale reconnue.

La libre circulation des expressions d'une langue à l'autre non seulement ressuscite le français « congolais » avec ses termes comme matabiches (pourboire) ou Radio-trottoir (rumeur) mais l'enrichit aussi « un sourire technique » et signe un permanent transfert de sens qui donne aux mots employés une valeur ajoutée à caractère anthropologique comme dans " un coup d'œil compliqué ». C'est dire combien dans ce pays où le mauvais œil est prégnant, un coup d'œil compliqué est déjà un avertissement. Et le corps qui le supporte cesse d'être un corps simple pour devenir un nœud de significations que l'écriture s'attache à symboliser dans les maux de l'Afrique postcoloniale.

\section{Sony, un moraliste rabelaisien}

Que le corps entretienne une relation privilégiée au pouvoir est la preuve qu'il est fait de chair, de viande et de sang. Trois termes par lesquels Sony Labou Tansi le décompose tout le long de son texte et par lesquels il désigne tout simplement la vie: «Mais ces choses c'est mon sang, c'est ma viande, c'est ma vie ${ }^{35}$. " L'exercice consiste alors à transposer en images saisissantes tous les états du corps dont les métaphores viennent emplir le texte de leur homologie à la vie ou à son effondrement : « Le boulot, oui. Mais ce corps n'est plus tout à fait le corps du boulot ${ }^{36}$. » Et cela, jusqu'à la démesure ; on dira alors de Sony Labou Tansi qu'il est le Rabelais africain.

De l'écriture rabelaisienne de Sony Labou Tansi, les critiques n'ont souvent retenu que le rire carnavalesque provoqué par les bouffonneries des pères de la nation de son roman La Vie et demie, ou de L'État honteux oubliant qu'elle est aussi faite de ce puissant souffle de vie qui traverse L'Anté-peuple, inédit tant par sa richesse lexicale que par sa

Continents manuscrits, 4 | 2015 
déclinaison grivoise et volontiers scatologique : «Il s'est "merdé" de vin au retrait de deuil du citoyen commissaire. Merdé, ce qu'on appelle merdé. Il a même "merdé" sur les gens. On l'a trimballé comme un bouchon de liège. Il puait ${ }^{37}$. » Ou plus directement : "Ce n'est pas le nombre de mouches qui fait la quantité de caca $^{38}$.» On comprend que dans cette déclinaison, le premier sens à être sollicité est l'odorat: «odeur des petits sous détournés du Trésor public ${ }^{39}$, odeur des voitures brûlées, odeur de la patronne, des cigarettes, du côté pourri des chambres du Magistrat. Tout y va de son remugle : le fleuve, le sang, le matelas du commissariat, les femmes, les hommes et même soi-même. Il n'y a pas meilleur instructeur sur une situation que l'odeur : « Elle était revenue. Mais elle sentait le Belge ${ }^{40}$. $\gg$ Ainsi, dans la lettre d'adieu que Yavelde écrit avant de se suicider, elle se dit rien moins que dépassée par sa propre odeur « Quand votre propre odeur vous dépasse (...) $»^{41}$. De même, on apprend à renouer avec soi-même en allant à la rencontre de ses odeurs perdues. Après quatre ans de prison, Dadou repart dans son ancienne maison dévastée pour retrouver ses odeurs : «Mais je suis resté quatre ans en arrière. Je me cherche. Il faut que je me cherche. Là j'ai rencontré mes odeurs. Tu ne peux pas savoir ${ }^{42}$.»

Mais le rapprochement avec Rabelais vaut aussi pour le retournement parodique du thème de l'enfer :

- Montrez-moi le chemin, dit Dadou.

- Pourquoi demandez-vous le chemin de l'enfer?

- Je connais un démon ${ }^{43}$.

Bien qu'il apparaisse comme l'expression privilégiée du négatif, l'enfer postcolonial, « route du deuil et de la désolation $»^{44}$ où résonnent les plaintes sourdes et continues de l'homme non coupable mais voué à un châtiment sans demi-mesures et sans fin, est aussi celui de son rachat. À condition que celui-ci transite par le don de soi, parce que «(...) parfois la plus grande chose qu'on puisse faire au monde passe par sa propre peau. Et on la fait ${ }^{45}$. »

Tchicaya U'Tamsi avait coutume de dire que Sony était un fieffé moralisateur. Je le pense également. Non seulement parce qu'il s'est attaché à la peinture des mœurs de la société politique congolaise mais parce qu'il est un spectateur du théâtre vivant du monde qu'il déchiffre à travers les proverbes connus, inventés ou détournés qui émaillent ses textes ou les aphorismes en tout genre qui ponctuent toujours chacune des réflexions de ces personnages. Citons en quelques exemples :

Le vide c'est la mère du désespoir. Dadou s'efforçait de ne pas désespérer. La vie même quand elle tomberait, il y croirait encore ${ }^{46}$.

C'est le bonheur des têtards. Maintenant, qui sait si vous serez grenouille ou crapaud $^{47}$ ?

(...) l'homme n'est beau que s'il connaît le prix du choix ${ }^{48}$

Le besoin des autres est une faiblesse ou simplement une duperie ${ }^{49}$.

Je considère le bonheur comme le premier devoir de l'homme sur cette terre, je reste toujours la même candidate au bonheur $(. . .)^{50}$.

On a toujours besoin d'un plus grand que soi (...).

Qui mange le matin évite de bâiller à l'enterrement ${ }^{51}$. 


\section{Empreinte historique et temps prophétiques} vision simple des choses à la complexité d'un monde où l'on peut voir disparaître ses capacités de discernement dans le flot des informations débitées. Il nous fait pénétrer dans une expérience cognitive spécifique de la situation postcoloniale, qui, au-delà des faits relatés, nous permet d'estimer les choses selon leur nature profonde. Cependant, la clairvoyance scripturaire de Sony Labou Tansi ne déduit rien, elle n'offre aucune orientation salvatrice même pas dans la rédemption meurtrière qu'il décrit. Est-ce d'ailleurs une rédemption? Tout juste permet-elle de préciser ces impressions initiales de cette vie mortifère en se basant sur des supports socio-anthropologiques bien ordonnés parmi lesquels la structure parentale occupe une place centrale puisqu'elle affecte jusqu'à la reproduction sociale dans ce pays : « où pour être quelque chose, il faut être cousin ou neveu de grands $\|^{54}$. Au centre de cette architecture socioanthropologique, une place importante revient à la figure du Blanc - même si elle n'est évoquée qu'en filigrane - dont la représentation irrigue encore l'imaginaire de ces anciens colonisés comme dans ce dialogue à la fin du chapitre VI entre Dadou et la patronne du Magistrat Pub :

-Vous payez comme un Blanc, je vous céderai à crédit.

- Vous avez une bonne opinion des Blancs.

- Tout le monde.

- S'ils étaient aussi pauvres que nous, les Blancs ne paieraient pas toujours.

- Il y a des Noirs plus riches que des Blancs et qui vous paient comme des Noirs ${ }^{55}$.

8 C'est que dans cet imaginaire, le Blanc est toujours le justificatif des situations improbables. Ainsi, la notoriété de l'oncle guérisseur de Dadou lui vient d'avoir soigné des Blancs : «S'il ne te guérit pas, disait l'opinion, c'est que le diable t'a mis du salé dans les pieds; et, dans le domaine vicieux de la stérilité, il avait soigné même des Blancs $^{56}$. » Bien que le héros s'en défende, tout vient lui rappeler que le fonctionnement du pays repose encore sur le retour même symbolique du colonisateur, comme l'état des chaussées mal entretenues "qui attendaient la prochaine visite du président français pour quémander aux autorités un autre rien de bitume (...) $\aleph^{57}$. Ainsi, le R. P. Van der Weldyck peut y aller de son couplet anti-indépendance : «C'est ça votre 
Afrique, c'est ça vos indépendances et vos révolutions d'Afrique : tout commence par les jambes. Il faut qu'on ouvre un ministère des jambes, vous y avez votre place. (...) Vous y venez à coups d'oncles, à coups de cousins (...) alors que votre don est dans les jupons ${ }^{58}$.»

Être du côté de la clairvoyance exige surtout du regard qui « voit les choses » qu'il soit restitué dans sa temporalité. Si Sony Labou Tansi est un voyant, c'est avant tout parce que l'auteur de L'Anté-peuple « voit demain avec des yeux d'aujourd'hui » comme l'avait déjà fait remarquer Sylvain Bemba. La parole du voyant engage un rapport au temps. Mais le temps dans lequel se déroule l'histoire, jour après jour, ne recouvre pas le temps du récit, il est une contraction du temps réel « Cinq ans étaient passés comme de l'eau ${ }^{59}$. » jusque dans la subjectivité des acteurs : « Le temps piétinait dans sa tête. Dans son ventre. Dans sa gorge. Comme un troupeau de jours et de nuits ${ }^{60}$.» Ou plus loin, « Il arrivait à 5 ou 6 nuits blanches avant d'en rencontrer une noire ${ }^{61}$. " D'un côté, le récit se périodise en fonction du temps des autres : «Il ne pouvait plus revenir. Une chose à faire, une seule : rester dans ce temps des autres qui bourdonnait autour de lui comme un nuage d'abeilles ${ }^{62}$. $\gg$ De l'autre, l'histoire se déroule en fonction d'une restauration du temps où le présent est soustrait pour être fédéré à l'annonce d'un impossible avenir.

C'est de cette vision que jaillit le pressentiment dans sa dimension prophétique - on dit de Sony qu'il est prophète - comme un long murmure intérieur sur une situation que vérifie chaque jour l'évolution de cet ensemble constitué par les deux Congo dont il est issu. Ce pressentiment, plusieurs années plus tard, garde toute son actualité car il s'alimente et se renouvelle, non sur une simple prémonition de ce qui s'y est déjà produit mais sur une véritable lecture d'empreinte historique. Cette empreinte s'observe dans la critique politique que contiennent les romans de Sony Labou Tansi. Dans L'Anté-peuple elle exhorte à la prise de distance, à la prudence comme dans ce constat: « Mais dans ces pays jeunes, les responsables, par prudence, il faut d'abord les prendre pour des voleurs. Ils avaient d'ailleurs tout fait pour acquérir cette réputation et s'efforçaient de la conserver ${ }^{63}$.»

31 Cette assertion, riche à plus d'un titre, l'est avant tout parce que la prudence est une disposition pratique, un principe (de précaution) requis contre les dangers du crédit aveugle accordé aux dirigeants africains ; des dirigeants dont les pratiques, en l'absence de certitudes de changements, devraient nous guérir de prévisibles désillusions. Par anticipation du scénario du pire.

Ensuite, c'est cette disposition qui devrait accompagner la distinction du vrai de la vérité, du réel de la réalité. Car elle enseigne de voir la réalité au-delà de la vérité immodérée de la loi et de ses apparences: «Une loi n'a d'yeux que pour voir les apparences. Et quand les apparences vous accusent, la loi s'en lave les mains ${ }^{64}$. " Le champ qu'ouvre alors Sony dans L'Anté-peuple est celui de la critique radicale de l'État postcolonial où les énoncés usuels autour de l'état de droit s'épuisent au contact d'une réalité qui fait corps avec la loi. Une loi niée parce que consubstantielle à la vérité du pouvoir, c'est-à-dire à ses apparences.

Il n'y a vérité, en effet, qu'en référence à un énoncé que nous jugeons conforme à la réalité ; or ici, l'énoncé est dans l'apparence et pourtant l'apparence n'est pas fausse, elle est juste authentique. Car dans ce qui est authentique, l'apparence est l'achèvement de la réalité. L'authenticité zaïroise est donc la fabrique légale de l'apparence postcoloniale comme réalité. Et celle-ci n'est pas dans la loi en tant que 
telle mais dans le rapport entre la loi, sa représentation et son application par ceux qui en ont reçu mandat : " les gendarmes (...) Ils sont aveugles comme la loi. Et comme elle, brutaux $^{65}$. " Rien ne protège donc contre la loi. "La seule possibilité d'échapper aux brutalités de la loi mesquine de l'uniforme c'est d'être grand - grand, c'est-à-dire grosse brute ${ }^{66}$. $»$ Etre au-dessus de la loi devient le seul moyen d'échapper à cette légalité mortifère. Et comme tout le monde cherche à se mettre sous la protection d'un plus grand que la loi, personne n'est plus à sa place : «Dadou se rappela une autre de ses lectures: l'Afrique, cette grosse merde où tout le monde refuse sa place. Un merdier, un moche merdier, ce monde ${ }^{67}$ !»

Le sens du désordre africain est là, dans cet étrange puzzle où rien ni personne n'est plus à sa place, même pas la loi. Et «Quand la loi a échappé des mains de ceux qui la contrôlent, elle devient une incomparable machine à tuer ${ }^{68}$. " Ceux qui ne peuvent pas avoir le bénéfice d'un parapluie, les plus nombreux, deviennent tout simplement minoritaires au regard de la loi et de ce fait perdent tous leurs droits : «Et ça me fait marrer qu'on parle de minorités blanches, alors que toute l'Afrique est inondée de minorités noires ${ }^{69}$. $\gg$ Coupables de ce qu'ils sont innocents et sans défense contre leur propre innocence, ils cessent d'être sûrs de leur vie. «C'est les innocents qu'on tue ${ }^{70}$." Mais mourir ne met pas à l'abri de la répression : «On a parfois arrêté des gens qui étaient morts $^{71}$.» Comble de l'absurdité, dirions-nous, mais qui octroie justement à l'écrivain sa place de prophète. Car il ne peut y avoir de prophète que là où il $\mathrm{y} a$ négation de la loi, d'où la nécessité de la restauration d'un temps où le présent suppute la fin des souffrances dans l'acte sacrificiel de Dadou.

\section{Conclusion}

La conclusion, je l'emprunte à un ami, Nicolas Martin-Granel : L'Anté-peuple est un roman plus philosophique que psychologique «Je parle d'un autre monde», plus existentiel qu'existentialiste Dadou se sent «inexister », où l'on pense, débat et se bat beaucoup, pas seulement Dadou mais aussi les pêcheurs, les maquisards, les femmes, et notamment Yavelde et Yealdara. Or, ces superbes héroïnes de tragédie - l'une donne sa vie pour Dadou, l'autre son corps - sont les deux faces du destin cynique des pays qui exigent qu'on les aime d'un amour qui tient de «l'acte de respirer»: «Ah! Putain de pays ! [dit en substance Dadou] on n'a même pas le cœur de le détester ${ }^{72}$.»

\section{NOTES}

1. Coffret L'atelier de Sony Labou Tansi, Nicolas Martin-Granel et Greta Rodriguez (dir.), Paris, éditions Revue noire, 2005.

2. Sony Labou Tansi, L'Anté-peuple, Paris, éditions du Seuil, 2010. Toutes les références des citations de l'ouvrage correspondent à cette édition.

3. http://motspluriels.arts.uwa.edu.au/MP1099slt.html

4. Sony Labou Tansi, L'Anté-peuple, Paris, éditions du Seuil, collection Points, 2010, p. 210. 
5. Ibid., p. 41.

6. Ibid., p. 172

7. Ibid., p. 169, 176, 179.

8. Ibid., p. 170.

9. Ibid., p. 22.

10. Ibid., p. 75.

11. Ibid., p. 39.

12. Ibid., p. 55.

13. Ibid., p. 13-14.

14. Ibid., p. 110.

15. Ibid., p. 105.

16. Ibid., p. 192.

17. Ibid., p. 120.

18. Ibid., p. 127.

19. Ibid., p. 35.

20. Ibid., p. 47.

21. Ibid., p. 47.

22. Ibid., p. 72.

23. Ibid., p. 161.

24. Ibid., p. 184.

25. Ibid., p. 131.

26. Ibid., p. 164.

27. Ibid., p. 196.

28. Ibid., p. 151.

29. Ibid., p. 203.

30. Ibid., p. 77.

31. Ibid., p. 41.

32. Ibid., p. 37.

33. Ibid., p. 60.

34. Ibid., p. 117.

35. Ibid., p. 116.

36. Ibid., p. 62.

37. Ibid., p. 44.

38. Ibid., p. 76.

39. Ibid., p. 34.

40. Ibid., p. 130.

41. Ibid., p. 77.

42. Ibid., p. 114.

43. Ibid., p. 140.

44. Ibid., p. 141.

45. Ibid., p. 154.

46. Ibid., p. 33.

47. Ibid., p. 39.

48. Ibid., p. 52.

49. Ibid., p. 109.

50. Ibid., p. 32.

51. Ibid., p. 133.

52. Ibid., p. 58.

53. Ibid., p. 71.

54. Ibid., p. 30. 
55. Ibid., p. 64.

56. Ibid., p. 30.

57. Ibid., p. 75.

58. Ibid., p. 65-66.

59. Ibid., p. 174.

60. Ibid., p. 95.

61. Ibid., p. 95.

62. Ibid., p. 107.

63. Ibid., p. 34.

64. Ibid., p. 80.

65. Ibid., p. 67.

66. Ibid., p. 67-68.

67. Ibid., p. 68.

68. Ibid., p. 150.

69. Ibid., p. 134.

70. Ibid., p. 179.

71. Ibid., p. 149.

72. Ibid., p. 124.

INDEX

Mots-clés : Sony Labou Tansi, L'Anté-peuple, Congo

\section{AUTEUR}

PATRICE YENGO

Université de Brazzaville, République du Congo 